\title{
Company Profile
}

\section{Moxtek, Inc.}

Moxtek empowers creators of cutting edge technology to break the barriers of scientific performance by engineering precision solutions developed through shared expertise.

Since we were founded in 1986, Moxtek has become a leading supplier of advanced nano-optical and X-ray components used in display electronics, imaging, and analytical instrumentation.

It is our goal is to become our customers' preferred supplier. To achieve customer satisfaction, we are committed to a culture of: compliance to internal and external requirements, continuous improvements, collaboration, and customer focus.

X-ray Windows

- AP Windows

- DuraBeryllium Windows

- ProLINE Windows

- Sealed Proportional Counter Windows

$\mathrm{X}$-ray Sources

- Soft X-ray Sources

- Side Window X-ray Sources

- End Window Cabled X-ray Sources

- Monoblock X-ray Sources

- X-ray Source Controllers

$\mathrm{X}$-ray Detectors

- Si-PIN Detectors

Ultra-Low Noise X-ray JFETs

Optics

- Visible Polarizers

- Reflective Normal Incidence Polarizers

- Absorptive Polarizers

- Polarizing Beam Splitters

- Broadband Polarizers

- Pixelated Polarizers

- UV Polarizers

- IR Polarizers

We are actively engaged in the development and manufacturing of innovative technology. We believe our future success lies in our collaboration with both current and future partners. Please contact us with any ideas you may have for new products or collaboration.

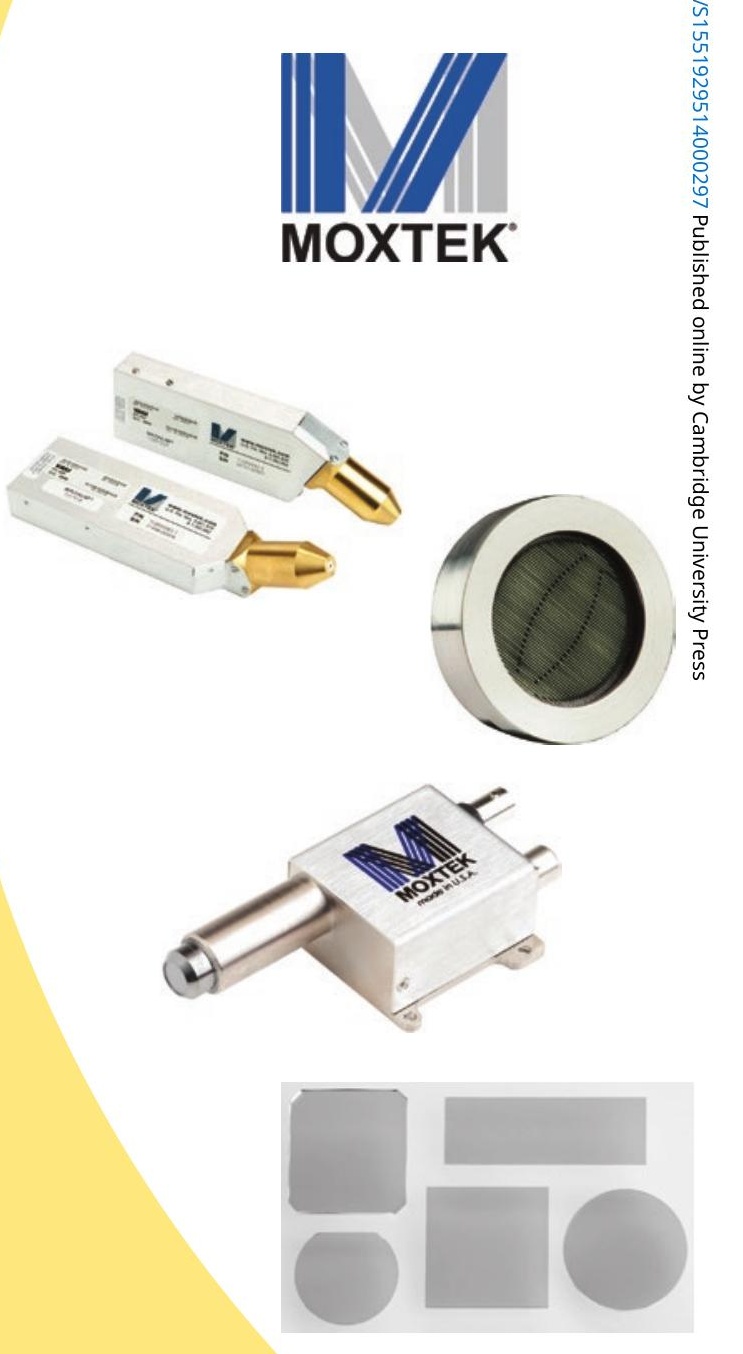

\section{How to find us}

Moxtek, Inc.

452 W. $1260 \mathrm{~N}$

Orem, UT 84057

Tel: 1-801-225-0930

Fax: 1-801-221-1121

Email: info@moxtek.com

www.moxtek.com

http://www.moxtek.com/contact

/distributors.html

Moxtek, Inc. is a subsidiary of Polatechno Co., Ltd., 192-6, Aza Shimogawara, Inamasu, Itakura-ku, Joetsu City, Niigata Prefecture 944-0101, Japan. 